\title{
Robust Vehicle Dynamics Control for a Sharp Curve With Uncertain Road Condition
}

\author{
Jing Miao ${ }^{1,2,3 *}$, Yifan Dai ${ }^{2}$, Ou Xie ${ }^{1}$, Hao Chen ${ }^{1}$, Fuzhou Niu ${ }^{1}$, Yehu Shen ${ }^{1}$, Yong Zhi Wu ${ }^{1}$, \\ Hui Sun ${ }^{2}$, Xuemei Niu ${ }^{1}$, Qixin Zhu ${ }^{1}$ and Wenjiang Shen ${ }^{3}$ \\ ${ }^{1}$ School of Mechanical Engineering, Suzhou University of Science and Technology, Suzhou, China, ${ }^{2}$ Suzhou Automotive \\ Research Institute, Tsinghua University, Suzhou, China, ${ }^{3}$ Suzhou Institute of Nano-Tech and Nano-Bionics(SINANO), Chinese \\ Academy of Sciences (CAS), Suzhou, China
}

OPEN ACCESS

Edited by:

Yahui Zhang,

Yanshan University, China

Reviewed by:

Xun Shen,

Tokyo University of Agriculture and

Technology, Japan Yang Tian,

Yanshan University, China

${ }^{*}$ Correspondence: Jing Miao

jmiao@mail.usts.edu.cn

Specialty section: This article was submitted to Wind Energy,

a section of the journal Frontiers in Energy Research

Received: 21 August 2021 Accepted: 08 September 2021 Published: 14 October 2021

Citation: Miao J, Dai Y, Xie O, Chen H, Niu F, Shen $Y$, Wu YZ, Sun H, Niu X, Zhu Q and Shen W (2021) Robust Vehicle Dynamics Control for a Sharp Curve

With Uncertain Road Condition.

Front. Energy Res. 9:762246.

doi: 10.3389/fenrg.2021.762246
Recently, more and more research has been conducted to develop Connected Autonomous Vehicles (CAVs) applications that ensures the safety driving of CAVs under some extreme situations. This brief presents a robust control strategy for CAVs to preserve a precise tracking performance and maintain the stability of lateral dynamics when passing a sharp curve with uncertain road friction coefficient changes. In the proposed robust lateral dynamics control, robust optimization-based lateral dynamics controller is designed to achieve the stability of the lateral dynamics with the consideration of the road friction coefficient uncertainty. Simulation validations are carried out to evaluate the proposed control strategy. The results show that the robust optimization-based lateral dynamics can improve the robustness even with the uncertainty of the road friction coefficient.

Keywords: model predictive conrol, robust optimisation, vehicle dynamic, uncertainty, stability

\section{INTRODUCTION}

Autonomous vehicles will meet more emergency scenarios when leaving the research laboratory and entering public roads (Kritayakirana and Gerdes, 2012; Shen and Raksincharoensak, 2021). Vehicle stabilization under uncertain scenarios is one of the most important issues in the control of autonomous vehicles (Yue et al., 2019; Shen et al., 2020a; Guo et al., 2020). Recently, Model Predictive Control (MPC) has been used to improve the vehicle dynamics stability (Yuan et al., 2019). In (Taghavifar, 2019), neural network autoregressive with exogenous input system has been applied to obtain an accurate and explicit model in order to contribute to the control of the system over the prediction horizon. (Weiskircher et al., 2017). proposed a MPC-based predictive trajectory guidance and tracking control framework for autonomous and semiautonomous vehicles in dynamic public traffic. Moreover, a data-driven predictive control is proposed in (Li and Schutter, 2021) which is model-free predictive control method.

However, the normal MPC without considering the uncertainty is not able to address the problem caused by environment uncertainty. The state space model-based prediction has large variance and even mean bias if there are any uncertainties in disturbance or the system parameters (Shen et al., 2020b). If there is uncertain road friction changes when passing a sharp curve and the model used in MPC cannot reflect the uncertainty, MPC will lose some precise on the lateral dynamics control. To improve the robustness against uncertainty, it is necessary to design a robust controller. In (Heshmati-Alamdari et al., 2020), a robust predictive controller is designed for underwater robotic vehicles which forms a high robust closed-loop system against parameter uncertainties. 


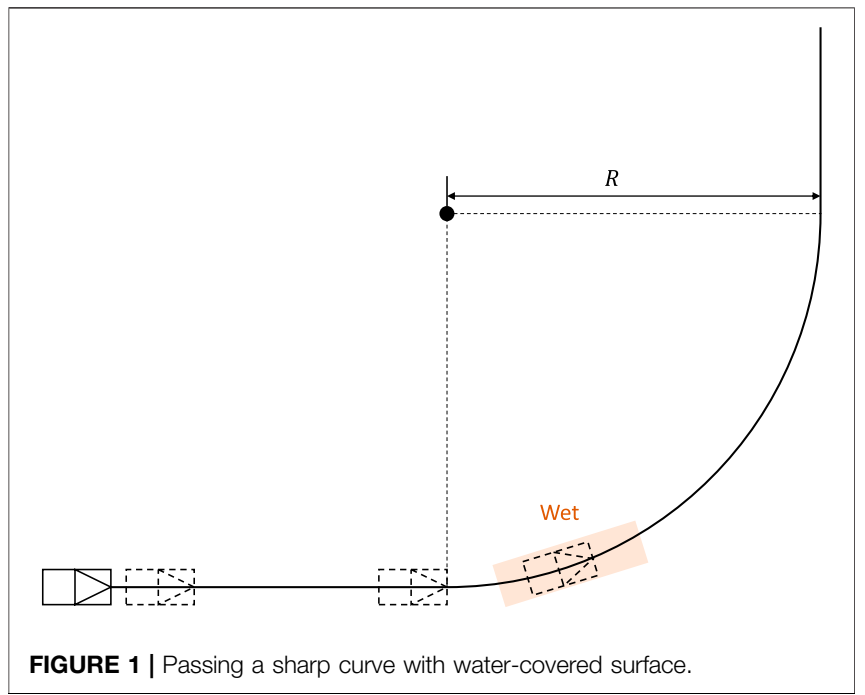

Besides, (Gao et al., 2021), proposed a robust lateral trajectory following control for autonomous vehicles. Robust model predictive control is a potential solution to the issue caused by uncertain road friction in this research. In the problem formulation of robust model predictive control, the road friction is regarded as a uncertain variable. For all possible realizations of uncertain variable, a fixed control law has a cost. We focused on finding a control law that minimize the upper bound of the cost for all possible realizations of uncertain variable. In this way, the robustness of the control strategy is able to be attained. To achieve robust model predictive controller, it is essentially to solve a robust optimization problem or a chance constrained optimization problem in every time step (Nemirovski and Shapiro, 2006; Shen et al., 2019). Although it is NP-hard to solve a robust optimization problem or a chance constrained optimization problem (Hong et al., 2011; Geletu et al., 2017; Pena-Ordieres et al., 2020), the approximate solution can be obtained by formulating a solvable approximate problem of the original one (Luedtke and Ahmed, 2008; Shen et al., 2021; Campi and Garatti, 2019, 2011). Robust model predictive control was widely applied in water qulity management (Takyi and Lence, 1999) and other process control applications (Henrion and Moller, 2003). Recently, robust model predictive control has been applied to the automotive powertrain control to optimize the fuel efficiency with stochastic constraint on the knock (Shen et al., 2017; Shen and Shen, 2017) and the energy management system in hybrid electric vehicle (Shen et al., 2016). Robust model predictive control can also be applied to ensure the robustness for an autonomous vehicle when it passes a sharp curve with uncertain road condition.

This paper presents a novel robust model predictive control strategy for automated vehicles to preserve a precise tracking performance and maintain the stability of lateral dynamics. The optimal feedback control input is obtained in every step by solving a robust optimization problem. The robust optimization problem is solved by scenario approach introduced in (Calariore and Campi, 2006). Simulation validations are carried out to evaluate the proposed control strategy.

\section{PROPOSED METHOD}

\subsection{Background and Problem Description}

In Figure 1, the vehicle passed a sharp curve with water-covered surface. The water-covered surface is the area with orange color. The single track model of vehicle dynamics can be described by the following equations:

$$
\begin{gathered}
\dot{y}_{c}=\dot{y}_{c}, \\
\ddot{y}_{c}=-\frac{2\left(C_{f}+C_{r}\right)}{m V} \dot{y}_{c}+\frac{2\left(C_{f}+C_{r}\right)}{m} \phi+\frac{2\left(l_{r} C_{r}-l_{f} C_{f}\right)}{m V} \dot{\phi} \\
+\frac{2 C_{f}}{m} \delta_{f}, \\
\dot{\phi}=\dot{\phi}, \\
\ddot{\phi}=\frac{2\left(l_{r} C_{r}-l_{f} C_{f}\right)}{I z V} \dot{y}_{c}+\frac{2\left(l_{f} C_{f}-l_{r} C_{r}\right)}{I z V} \phi-\frac{2\left(l_{f}^{2} C_{f}+l_{r}^{2} C_{r}\right)}{I z V} \dot{\phi} .
\end{gathered}
$$

Here, $y_{c}$ is the lateral distance. $\dot{\phi}$ is the yaw rate. $m$ is the mass of the vehicle. $\delta_{f}$ is the steer angle. $V$ is the vehicle speed.

In order to apply MPC, the vehicle lateral dynamics model is transformed to the lateral deviation from the reference model. The used linear model is as

$$
\left[\begin{array}{l}
\dot{y}_{c r} \\
\ddot{y}_{c r} \\
\dot{\phi}_{c r} \\
\ddot{\phi}_{c r}
\end{array}\right]=A\left[\begin{array}{c}
y_{c r} \\
\dot{y}_{c r} \\
\phi_{c r} \\
\cdot \phi_{c r}
\end{array}\right]+B \delta_{f}+C \frac{1}{R}
$$

Where

$$
\begin{aligned}
& A=\left[\begin{array}{cccc}
0 & 1 & 0 & 0 \\
0 & -\frac{2\left(C_{f}+C_{r}\right)}{m V} & \frac{2\left(C_{f}+C_{r}\right)}{m} & \frac{2\left(l_{r} C_{r}-l_{f} C_{f}\right)}{m V} \\
0 & 0 & 0 & 1 \\
0 & \frac{2\left(l_{r} C_{r}-l_{f} C_{f}\right)}{I_{z} V} & \frac{2\left(l_{f} C_{f}-l_{r} C_{r}\right)}{I_{z} V} & -\frac{2\left(l_{f}^{2} C_{f}+l_{r}^{2} C_{r}\right)}{I_{z} V}
\end{array}\right] \\
& B=\left[\begin{array}{c}
0 \\
\frac{2 C_{f}}{m} \\
0 \\
\frac{2 l_{f} C_{f}}{m}
\end{array}\right] \\
& C=\left[\begin{array}{c}
0 \\
-V^{2}+\frac{2 l_{r} C_{r}-2 l_{f} C_{f}}{m} \\
0 \\
-\frac{2 l_{f}^{2} C_{f}+2 l_{r}^{2} C_{r}}{I z}
\end{array}\right]
\end{aligned}
$$




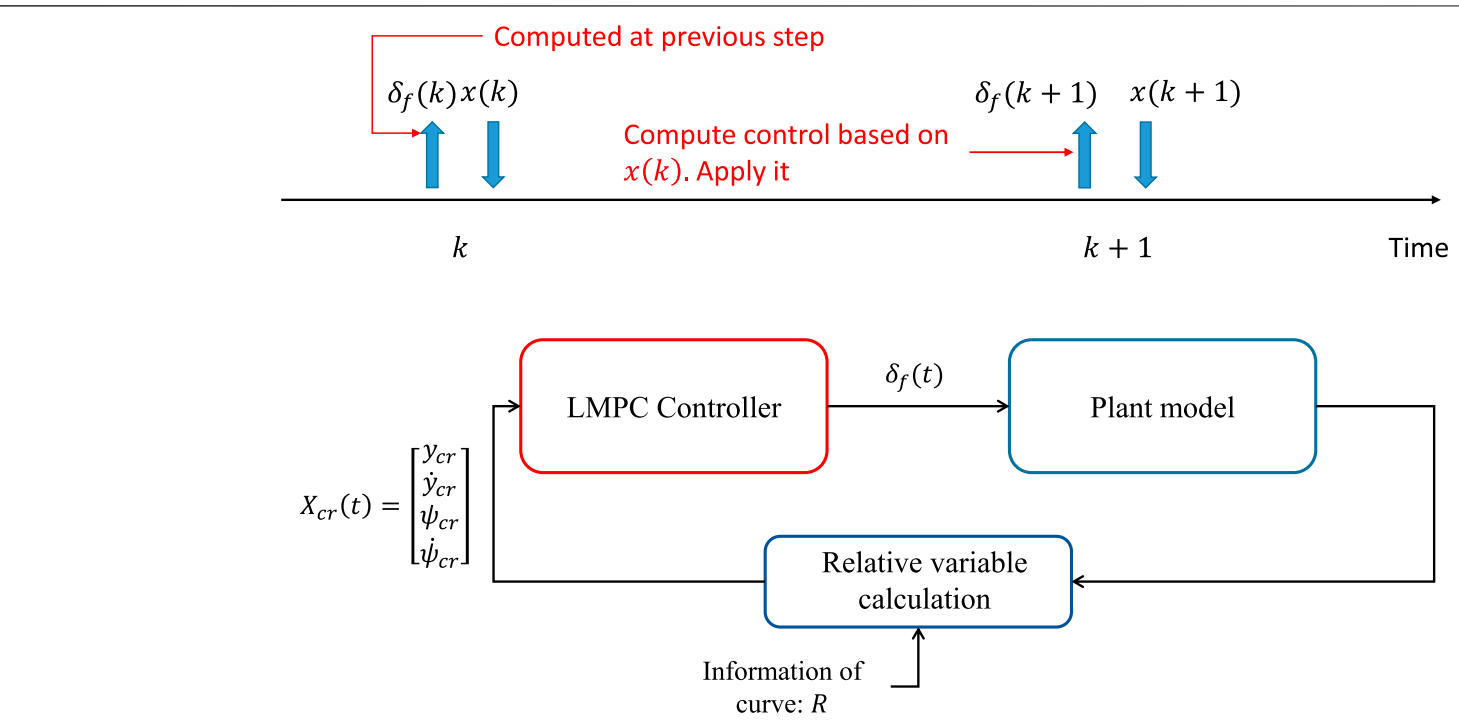

FIGURE 2 | Implementation of robust MPC.

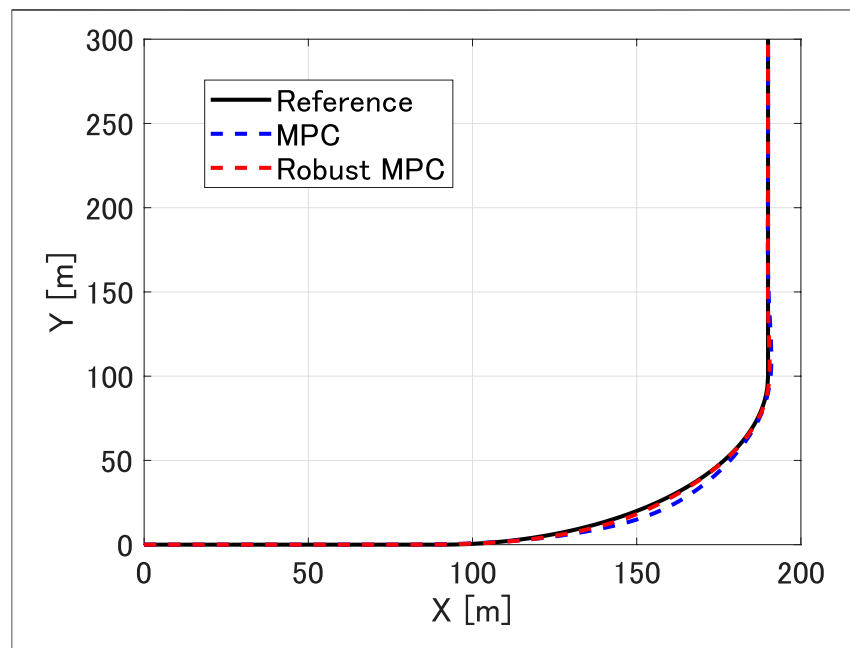

FIGURE 3 | Validation result.

Here, $y_{c r}$ is the lateral deviation from the reference trajectory. $\dot{\phi}_{c r}$ is the yaw rate. $R$ is the radius of the curve. $m$ is the mass of the vehicle. $\delta_{f}$ is the steer angle. $V$ is the vehicle speed.

Notice that $C_{f}$ and $C_{r}$ are both decided by the road friction coefficient. Since the road friction coefficient is uncertain, $C_{f}$ and $C_{r}$ are both uncertain variable as well.

Equation 5 is a continuous differential equation and can be transformed to a discrete state-space model by Euler method. Since at every time step, the state variable is decided by the input $\delta_{f}$ and the state variable in the previous step. The state variable at $k+1$ can be expressed by the previous input sequence $\delta_{f}(0), \ldots, \delta_{f}(k)$ and the state variable at the initial step. Since the objective is to minimize the difference between the actual trajectory and the reference one, the cost function is a function of the input sequence and known state variable at initial step. To obtain the optimal input, a robust optimization problem should be solved. The problem can be formulated generally by

$$
\begin{aligned}
& \min _{u \in \mathcal{U} \subset \mathbf{R}^{n_{u}}} J(u) \\
& \text { s.t. } h(u, \delta) \leq 0, \delta \in \Delta \subset \mathbf{R}^{n_{\delta}} .
\end{aligned}
$$

Here, $u=\left[\delta_{f}(0), \ldots, \delta_{f}(K-1), E\right]^{T} \quad$ if $\quad$ we consider $K$ steps forward. $\delta$ is the uncertain variable. In our problem, it includes $C_{f}$ and $C_{r} . J(u)=E$ and $h(u, \delta)$ is defined as

$$
\sum_{k=1}^{K} y_{c r}(k)-E
$$

\subsection{Scenario Approach}

In scenario approach, independent samples $\delta^{(i)}, i=1, \ldots, N$ is identically extracted from $\Delta$ randomly, a deterministic convex optimization problem can be formed as (Calariore, 2017; Campi et al., 2018; Campi and Garatti, 2018)

$$
\begin{aligned}
& \min _{u \in \mathcal{U} \subset \mathbf{R}^{n_{u}}} J(u) \\
& \text { s.t. } \quad h\left(u, \delta^{(i)}\right) \leq 0, \quad i=1, \ldots, N
\end{aligned}
$$

which is a standard finitely constrained optimization problem. The optimal solution $\hat{u}_{N}$ of the program Eq. 11 is called as the scenario solution for program Eq. 9 generally. Moreover, since the extractions $\delta^{(i)}, i=1, \ldots, N$ is randomly chosen, the optimal solution $\hat{u}_{N}$ is random variable. If $\hat{u}_{N}$ is expected to satisfy

$$
\operatorname{Pr}^{N}\left\{\left(\delta^{(1)}, \ldots \delta^{(N)} \in \Delta^{N}: V\left(\hat{u}_{N}\right) \leq \alpha\right\} \geq 1-\beta, \beta \in(0,1),\right.
$$



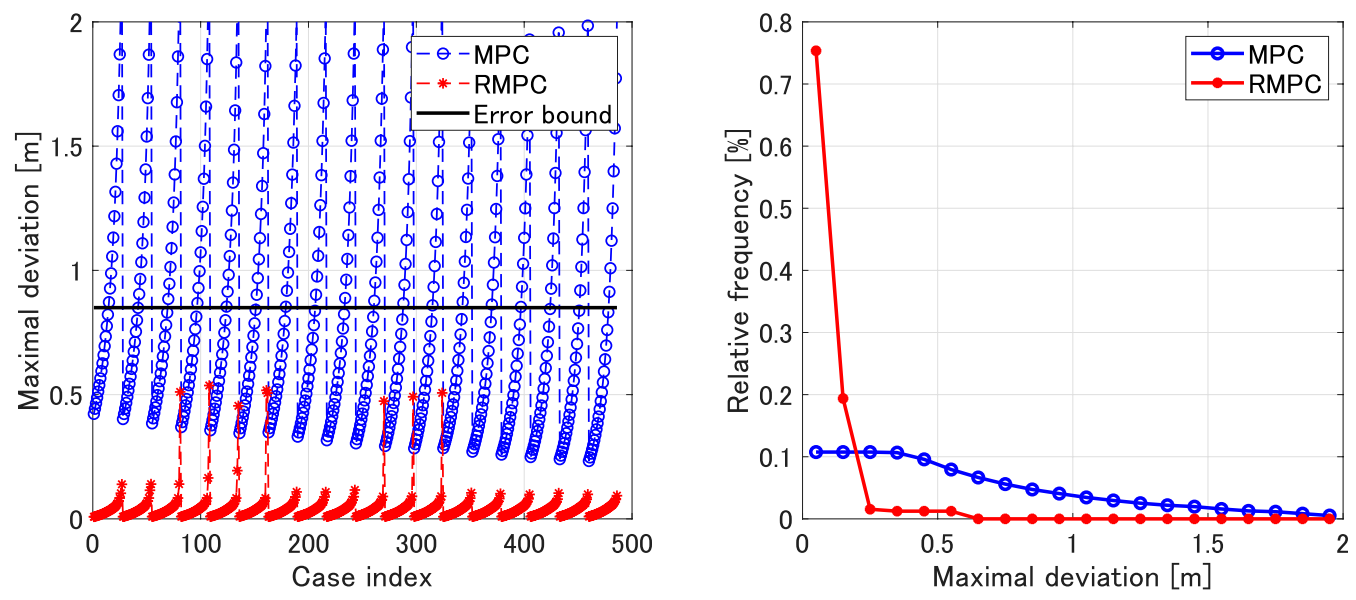

FIGURE 4 | Validation result.

then, $N$ should have a lower limitation $N_{l}$

$$
N \geq \frac{2}{\alpha} \ln \frac{1}{\beta}+2 n_{u}+\frac{2 n_{u}}{\alpha} \ln \frac{2}{\alpha} .
$$

Note that $\beta$ is an important factor and choosing $\beta=0$ makes $N_{l}=\infty$. Namely, if the number of chosen samples gets larger, the probability of satisfying the original probabilistic constraints approaches 1 . Actually, when number of chosen samples becomes infinity, the samples cover the whole sample space. The feasible area determined by probabilistic constraints is only a subset of whole sample space. Then, it becomes a problem which requires total robustness. Therefore, the scenario approach conducts to a solution with total robustness which is more conservative than the probabilistic constraints require.

\subsection{Implementation of Robust Model Predictive Control}

The implementation of robust MPC is shown in Figure 2. At time step $k+1$, it uses the first element of $u$ calculated in time step $k$ as the input. Namely, $\delta_{f}(k)=u(1) . x(k)$ denotes the state variable vector at time step $k$. Moreover, since the LMPC controller takes relative variable calculation as feedback, there will be a relative variable calculation. In the relative variable calculation, the relative variable is calculated based on the feedback state variable from plant model or real vehicle and the information of curve, for example, radius value $R$.

\section{VALIDATION RESULTS AND CONCLUSTION}

The validation is implemented by simulation. Since the real vehicle is not available, a plant model is established and used instead of the real vehicle. The plant model adopts the single track nonlinear model described by

$$
\begin{gathered}
\dot{v}_{x}-v_{y} \dot{\phi}=\frac{1}{m}\left(F_{f}^{x_{T}} \cos \delta_{f}+F_{r}^{x_{T}}-F_{f}^{y_{T}} \sin \delta_{f}\right), \\
\dot{v}_{y}+v_{x} \dot{\phi}=\frac{1}{m}\left(F_{f}^{y_{T}} \cos \delta_{f}+F_{r}^{y_{T}}+F_{f}^{x_{T}} \sin \delta_{f}\right), \\
I_{z z} \ddot{\phi}=l_{f} F_{f}^{y_{T}} \cos \delta_{f}-l_{r} F_{r}^{y_{T}}+l_{f} F_{f}^{x_{T}} \sin \delta_{f}, \\
{\left[\begin{array}{c}
\dot{p}_{x} \\
\dot{p}_{y}
\end{array}\right]=\mathcal{R}(\phi)\left[\begin{array}{c}
v_{x} \\
v_{y}
\end{array}\right] .}
\end{gathered}
$$

The magic formula is used to model the friction forces which refers to (Yuan et al., 2019).

For the simulation conditions, the radius has six options: $100,110,120,130,140$, and $150 \mathrm{~m}$. For each $R$, three coefficients of friction for the wet road is randomly generated from $(0.4,0.6)$. For each pair of a value of $R$ and a value of coefficients of friction, the following longitudinal velocity values have be tested:

$$
[0.4,0.42, \ldots, 0.92] \times \sqrt{R \mu_{w e t} g} .
$$

Figure 3 shows one example of the validation results. The friction coefficient of dry road is $\mu_{d r y}=0.8$ which the one of wet road is $\mu_{\text {wet }}=0.5$. The radius of the curve is $100 \mathrm{~m}$. The middle part of the road is wet. The longitudinal velocity for passing the curve is $V=65 \mathrm{~km} / \mathrm{h}$. If MPC is used by setting $C_{r}$ and $C_{f}$ according to $\mu_{d r y}=0.8$, the tracking error increases during the wet road. However, by considering $\mu \in[0.4,0.9]$, the robust MPC keeps the tracking performance stable during the wet road.

Figure 4 shows a comprehensive statistical validation results of all cases. Obviously, the robust MPC succeeded to decrease the maximal deviation into the error bound. However, the normal MPC failed in most cases since the model has a very large bias compared to the real dynamics due to the uncertain friction coefficient. 


\section{DATA AVAILABILITY STATEMENT}

The original contributions presented in the study are included in the article/Supplementary Material, further inquiries can be directed to the corresponding author.

\section{AUTHOR CONTRIBUTIONS}

All authors listed have made a substantial, direct, and intellectual contribution to the work and approved it for publication.

\section{REFERENCES}

Calariore, G. C., and Campi, M. C. (2006). The Scenario Approach Torobust Control Design. IEEE Trans. Automatic Control. 51 (5), 742-753. doi:10.1109/ tac. 2006.880951

Calariore, G. (2017). Repetitive Scenario Design. IEEE Trans. Automatic Control. 62 (3), 1125-1137. doi:10.1109/tac.2017.2788139

Campi, M. C., and Garatti, S. (2011). A Sampling-And-Discarding Approach to Chance-Constrained Optimization: Feasibility and Optimality. J. Optimization Theor. Appl. 148 (2), 257-280. doi:10.1007/s10957-010-9754-6

Campi, M. C., and Garatti, S. (2019). Introduction to the Scenario Approach. Philadelphia: MOS-SIAM Series on Optimization.

Campi, M. C., Garatti, S., and Ramponi, F. A. (2018). A General Scenario Theory for Nonconvex Optimization and Decision Making. IEEE Trans. Automat. Contr. 63 (12), 4067-4078. doi:10.1109/tac.2018.2808446

Campi, M. C., and Garatti, S. (2018). Wait-and-Judge Scenario Optimization. Math. Program 167, 155-189. doi:10.1007/s10107-016-1056-9

Gao, H., Kan, Z., and Li, K. (2021). Robust Lateral Trajectory Following Control of Unmanned Vehicle Based on Model Predictive Control. Ieee/asme Trans. Mechatron., early access. doi:10.1109/TMECH.2021.3087605

Geletu, A., Hoffmann, A., Klöppel, M., and Li, P. (2017). An Inner-Outer Approximation Approach to Chance Constrained Optimization. SIAM J. Optim. 27, 1834-1857. doi:10.1137/15m1049750

Guo, N., Zhang, X., Zou, Y., Lenzo, B., and Zhang, T. (2020). A Computationally Efficient Path-Following Control Strategy of Autonomous Electric Vehicles with Yaw Motion Stabilization. IEEE Trans. Transp. Electrific. 6 (2), 728-739. doi:10.1109/tte.2020.2993862

Henrion, R., and Moller, A. (2003). Optimization of a Continuous Distillation Process under Random Inflow Rate. Comput. Math. Appli. 45, 13-30. doi:10.1016/s0898-1221(03)80017-2

Heshmati-Alamdari, S., Karras, G. C., Marantos, P., Kyriakopoulos, K. J., and Kyriakopoulos, K. J. (2020). A Robust Predictive Control Approach for Underwater Robotic Vehicles. IEEE Trans. Contr. Syst. Technol. 28 (6), 2352-2363. doi:10.1109/tcst.2019.2939248

Hong, L. J., Yang, Y., and Zhang, L. (2011). Sequential Convex Approximations to Joint Chance Constrained Programs: A Monte Carlo Approach. Operations Res. 59, 617-630. doi:10.1287/opre.1100.0910

Kritayakirana, K., and Gerdes, J. C. (2012). Autonomous Vehicle Control at the Limits of Handling. Ijvas 10 (4), 271-296. doi:10.1504/ijvas.2012.051270

Li, D., and De Schutter, B. (2021). Distributed Model-Free Adaptive Predictive Control for Urban Traffic Networks. IEEE Trans. Contr. Syst. Technol., 1-13. early access. doi:10.1109/TCST.2021.3059460

Luedtke, J., and Ahmed, S. (2008). A Sample Approximation Approach for Optimization with Probabilistic Constraints. SIAM J. Optim. 19 (2), 674-699. doi:10.1137/070702928

Nemirovski, A., and Shapiro, A. (2006). Convex Approximations of Chance Constrained Programs. SIAM J. Optimization 17, 969-996. doi:10.1137/050622328

Peña-Ordieres, A., Luedtke, J. R., and Wächter, A. (2020). Solving ChanceConstrained Problems via a Smooth Sample-Based Nonlinear Approximation. SIAM J. Optim. 30 (3), 2221-2250. doi:10.1137/19m1261985

Shen, X., Ouyang, T., Yang, N., and Zhuang, J. (2021). Sample-Based Neural Approximation Approach for Probabilistic Constrained Programs. IEEE Trans. Neural Netw. Learn. Syst., 1-8. early access. doi:10.1109/TNNLS.2021.3102323

\section{FUNDING}

The authors appreciate the supports of Foundation of Natural Science Research in Colleges and Universities of Jiangsu Province (Grant: 18KJB510043), National Key R\& D Program of China (Grant: 2018YFB0105201), Natural Science Foundation of China (Grant: 51975394; 61903269) and Natural Science Foundation of Jiangsu Province (Contract: BK20200271).

Shen, X., Ouyang, T., Zhang, Y., and Zhang, X. (2020a). Computing Probabilistic Bounds on State Trajectories for Uncertain Systems. IEEE Trans. Emerg. Top. Comput. Intell., 1-6. early access. doi:10.1109/TETCI.2020.3019040

Shen, X., and Raksincharoensak, P. (2021). Pedestrian-Aware Statistical Risk Assessment. IEEE Trans. Intell. Transport. Syst. 22, 1-9. early access. doi:10.1109/TITS.2021.3074522

Shen, X., and Shen, T. (2017). Real-Time Statistical Learning-Based Stochastic Knock Limit Control for Spark-Ignition Engines. Appl. Therm. Eng. 127, 1518-1529. doi:10.1016/j.applthermaleng.2017.08.150

Shen, X., Zhang, J., and Shen, T. (2016). "Real-time Scenario-Based Stochastic Optimal Energy Management Strategy for HEVs," in Proceedings of 2016 European Control Conference, Aalborg, Denmark, 29 June-1 July 2016, 631-636. doi:10.1109/ecc.2016.7810359

Shen, X., Zhang, X., and Raksincharoensak, P. (2020b). Probabilistic Bounds on Vehicle Trajectory Prediction Using Scenario Approach. IFAC-PapersOnline 53 (2), 2385-2390. doi:10.1016/j.ifacol.2020.12.038

Shen, X., Zhang, Y., Shen, T., and Khajorntraidet, C. (2017). Spark advance SelfOptimization with Knock Probability Threshold for Lean-Burn Operation Mode of SI Engine. Energy 122, 1-10. doi:10.1016/j.energy.2017.01.065

Shen, X., Zhuang, J., and Zhang, X. (2019). Approximate Uncertain Program. IEEE Access 7, 182357-182365. doi:10.1109/access.2019.2958621

Taghavifar, H. (2019). Neural Network Autoregressive with Exogenous Input Assisted Multi-Constraint Nonlinear Predictive Control of Autonomous Vehicles. IEEE Trans. Veh. Technol. 68 (7), 6293-6304. doi:10.1109/tvt.2019.2914027

Takyi, A. K., and Lence, B. J. (1999). Surface Water Quality Management Using a Multiple-Realization Chance Constraint Method. Water Resour. Res. 35, 1657-1670. doi:10.1029/98wr02771

Weiskircher, T., Wang, Q., and Ayalew, B. (2017). Predictive Guidance and Control Framework for (Semi-)Autonomous Vehicles in Public Traffic. IEEE Trans. Contr. Syst. Technol. 25 (6), 2034-2046. doi:10.1109/tcst.2016.2642164

Yuan, H., Sun, X., and Gordon, T. (2019). Unified Decision-Making and Control for Highway Collision Avoidance Using Active Front Steer and Individual Wheel Torque Control. Vehicle Syst. Dyn. 57 (8), 1188-1205. doi:10.1080/ 00423114.2019.169745610.1080/00423114.2018.1535125

Yue, M., Ning, Y., Zhao, X., and Zong, G. (2019). Point Stabilization Control Method for WIP Vehicles Based on Motion Planning. IEEE Trans. Ind. Inf. 15 (6), 3368-3378. doi:10.1109/tii.2018.2875048

Conflict of Interest: The authors declare that the research was conducted in the absence of any commercial or financial relationships that could be construed as a potential conflict of interest.

Publisher's Note: All claims expressed in this article are solely those of the authors and do not necessarily represent those of their affiliated organizations, or those of the publisher, the editors and the reviewers. Any product that may be evaluated in this article, or claim that may be made by its manufacturer, is not guaranteed or endorsed by the publisher.

Copyright (c) 2021 Miao, Dai, Xie, Chen, Niu, Shen, Wu, Sun, Niu, Zhu and Shen. This is an open-access article distributed under the terms of the Creative Commons Attribution License (CC BY). The use, distribution or reproduction in other forums is permitted, provided the original author(s) and the copyright owner(s) are credited and that the original publication in this journal is cited, in accordance with accepted academic practice. No use, distribution or reproduction is permitted which does not comply with these terms. 